\title{
Factores clave en el proceso de adquisición de libros electrónicos
}

\author{
Por Juan-Carlos Martín-González y Emma Pivetta
}

\begin{abstract}
Resumen: Tras la implantación de las colecciones de revistas electrónicas en las bibliotecas académicas el siguiente paso es la ampliación de las colecciones de monografías con nuevos materiales en formato digital. La industria editorial internacional ofrece los contenidos de sus monografías en formato digital. En el presente artículo se revisan los factores clave a tener en cuenta en la fase de selección y adquisición de libros electrónicos así como la oferta editorial internacional existente en la actualidad. Así mismo se muestra una comparativa de los agregadores de libros electrónicos con mayor presencia en España.
\end{abstract}

Palabras clave: Libro electrónico, Contenidos electrónicos, Industria editorial, Editores internacionales, Modelos de adquisición, Desarrollo de colecciones.

\section{Title: Key factors for e-books acquisition}

Abstract: After the introduction of electronic journals collections in academic libraries the next step is to expand the existing collections of books and reference materials with new content in digital format. The international publishing industry is currently offering their books in digital format. The present article reviews the key factors to be considered in the selection and acquisition stages of electronic books as well as the existing international publishing offer. It is shown also a comparison of electronic books aggregators with strong presence in Spain.

Keywords: Electronic book, Electronic content, Publishing industry, International publishers, Acquisition models, Collection development.

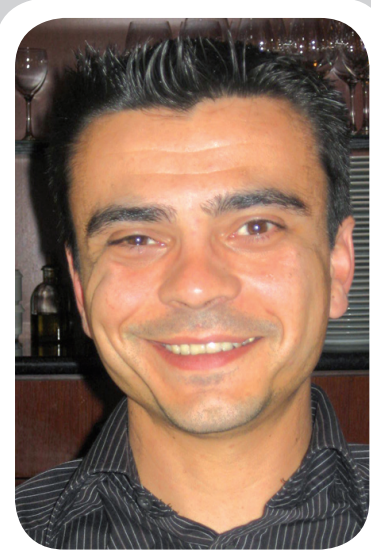

Juan Carlos Martín González, licenciado en Documentación por la Universidad de Salamanca, es actualmente Country Manager de Swets en España, después de haber ocupado diferentes posiciones dentro del equipo comercial de Swets. Ha publicado varios artículos relacionados con aspectos comerciales de las revistas electrónicas y la industria editorial así como intervenciones en diferentes congresos sobre información científico-técnica y perfiles profesionales de titulados en documentación.

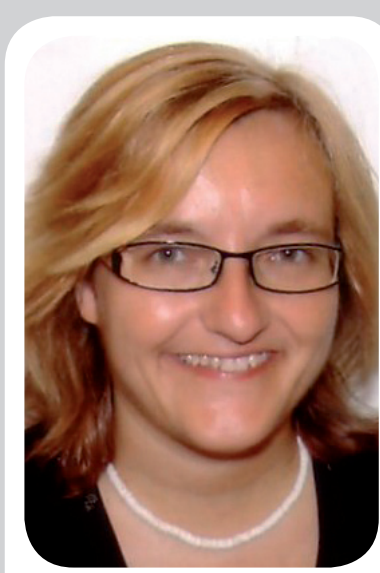

Emma Pivetta, licenciada en Sociología por la Fac. de Económicas de la Univ. de Barcelona (UB) y postgraduada en Proyectos Europeos por la Fundación Bosch y Gimpera, es responsable de Productos Electrónicos Swets en España. Fue técnico de proyectos de internacionalización del Consorcio de Promoción Comercial de Catalunya, responsable de la gestión de proyectos para la Secretaría general de Juventud de la Generalitat de Catalunya, así como investigadora y docente de la Cátedra Jean Monnet de Integración Europea de la UB.

Martín-González, Juan-Carlos; Pivetta, Emma. "Factores clave en el proceso de adquisición de libros electrónicos". En: El profesional de la información, 2008, julio-agosto, v. 17, n. 4, pp. 408-413.

DOI: 10.3145/epi.2008.jul.06

\section{Introducción}

LA EVOLUCIÓN DE LAS COLECCIONES digitales en el panorama de la biblioteca universitaria española durante los últimos 10 años ha sido vertiginosa, pues cuenta en la actualidad con cerca de $\mathbf{1 6 , 5}$ millones de documentos descargados desde recursos digitales de pago o bajo licencia, según datos estadísticos Rebiun 2007.

Inicialmente las revistas electrónicas han sido el principal objetivo de las bibliotecas españolas en su migración a los nuevos formatos.
Y dicho objetivo ha sido de sobra cubierto pues como prueba tenemos la media de 11.522 revistas electrónicas de pago suscritas por parte de los miembros de Rebiun.

El siguiente paso lógico en el desarrollo de las colecciones digitales es poner a disposición de profesores, investigadores y alumnos colecciones de monografías y obras de referencia en formato electrónico (que en este artículo llamamos eBooks). Esta fase ya ha comenzado y lleva un ritmo vivo gracias a las experiencias pasadas en las eta- pas de las colecciones de revistas electrónicas. El crecimiento de las monografías electrónicas entre los miembros de Rebiun ha experimentado un crecimiento del $37 \%$ ente 2005 y 2007 , pasando de 2.700 .000 a 3.695.000 e-libros accesibles por pago.

Tal y como sucediera en los primeros pasos de las revistas electrónicas nos encontramos en una fase de incertidumbre ya que a pesar de las similitudes entre libros y revistas, especialmente en el origen y contenidos de ambos, las diferen- 
cias son palpables y los modelos de adquisición e incluso acceso a los libros electrónicos presentan características propias.

En el presente artículo buscamos ofrecer una visión general de la oferta de libros electrónicos que las grandes editoriales internacionales dedicadas al sector académico y científico-técnico están proponiendo globalmente, haciendo un breve análisis de los puntos a tener en cuenta a la hora de adquirir y acceder a los mismos.

\section{2. ¿Dónde estamos?}

¿Qué modelos de libros-e hay actualmente en el mercado? ¿Cómo se distribuyen? ¿Qué soportes necesitan?

En trabajos existentes se tiende a categorizar el libro electrónico en base a cuatro modelos de acceso:

- acceso online,

- acceso offline,

- descarga y lectura en dispositivos específicos para la lectura de libros, y

- descarga y lectura en dispositivos genéricos.

Por acceso online se entiende aquel que se realiza a través del navegador de internet. Esta modalidad tiene como característica principal permitir al usuario final no sólo la lectura sino el enlace a otros recursos relacionados con la temática del libro electrónico, su autor, etc.

El acceso offline es la descarga a pc (o mac) de los contenidos de un libro electrónico desde un servicio suministrador de libros-e.

En el presente artículo nos centraremos en el libro electrónico dirigido a la investigación, el cual se encuadra dentro de la categoría de acceso online desde dispositivos genéricos.

Los eBooks tienen enormes ventajas para el ámbito de la investigación y el desarrollo en el sec-
"Este artículo se centra en el libro electrónico dirigido a la investigación, al cual se accede online desde equipos genéricos"

tor académico y científico-técnico, muy especialmente por presentar los siguientes beneficios:

\section{Ventajas de los libros-e}

\section{- Para los bibliotecarios}

- Ahorro de espacio en las estanterías de las bibliotecas

- No hay riesgo de que se dañen o sean robados

- Menor coste

- Posibilidad de obtener estadísticas de uso

- Registros Marc21

\section{- Para los usuarios}

- Disponibilidad horaria total: 24 horas al día, durante 7 días a la semana

- Funcionalidades multimedia

- Posibilidad de acceso de varios usuarios simultáneamente

- No es necesario esperar para acceder a su contenido

- Búsqueda avanzada

- Creación de notas y preferidos, y posibilidad de impresión selectiva.

\section{Variables a tener en cuenta en las políticas de acceso a eBooks}

El eBook no es el gemelo del libro impreso. No es la copia de un libro en formato papel pasado al formato digital, sino que es una herramienta de suma utilidad que ofrece infinidad de posibilidades, especialmente indicadas para la investigación y para la gestión diaria de las bibliotecas.
En este apartado se describirán las principales condiciones aplicadas al sector científico-técnico y académico, obviando las dispuestas para el acceso a libros-e con carácter más generalista y destinados el gran público.

Las principales variables a tener en cuenta son:

- Opciones de adquisición y licencias

- Requisitos técnicos (dispositivos, programas y método de autenticación)

- Temas y colecciones

- DRM's (digital rights management)

\subsection{Opciones de adquisición y licencia}

Pueden categorizarse en base a tres variables principales: modelo de adquisición, contenidos contratados y número de usuarios simultáneos.

El gráfico 1 da una idea resumida de los modelos de adquisición de eBooks.

Los principales modelos son:

- compra a perpetuidad, por la cual la institución adquiere en propiedad el contenido seleccionado, manteniendo los derechos de acceso sobre el mismo de forma indefinida; y

- suscripción, por la que la institución obtiene derechos sobre el material contratado y sus actualizaciones, o incluso en algunos casos sobre nuevas ediciones y nuevas obras añadidas a colecciones, durante un periodo concreto de tiempo y contra el pago de una cuota anual.

Solamente la editorial John Wiley ofrece un modelo híbrido denominado flexi-subscription, que permite la adquisición en propiedad del contenido contratado después de un periodo de tres años ininterrumpidos de suscripción. 


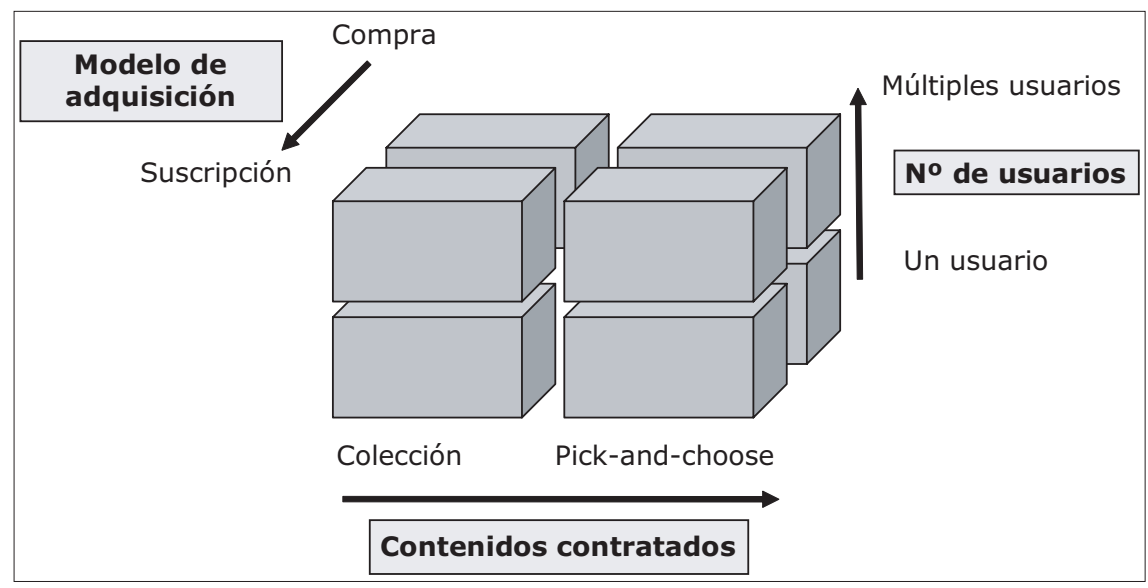

Gráfico 1. Modelo de adquisición de ebooks

Fuente: Maxim Van Gisbergen, Global product manager de Swets.

La adquisición de colecciones, bien sean acotadas temáticamente $o$ bien catálogos completos de editores, o la compra de obras de modo individual (conocido en el ámbito anglosajón como modelo Pick \& choose), varía en función de los contenidos contratados.

El último factor determinante en la adquisición de eBooks es el número de usuarios simultáneos. Actualmente la mayoría de grandes editoriales ofrece al acceso ilimitado en cuanto al número de usuarios simultáneos, lo que representa una gran ventaja frente al formato impreso. Solamente existen algunas excepciones, como por ejemplo Taylor \& Francis $(T \& F)$, que diferencia el número de usuarios simultáneos según el modelo de adquisición seleccionado.

\subsection{Requisitos técnicos}

Por norma general, para acceder a un eBook sólo es necesario disponer de un navegador y del programa Adobe Acrobat Reader, ambos gratuitos. Esto es así para las editoriales $C A B$, Cambridge University Press, Karger eBooks, RSC Publishing, Springer eBooks, Wiley eBooks y World Scientific eBooks. Sólo MedicinesComplete (de RPS Publishing) precisa que el navegador acepte cookies y, a ser posible, que soporte JavaScript, pero ésta es una excepción dentro de la norma general establecida.

Otro tipo de acceso se hace mediante equipos genéricos (como PDA o Palm) que permite, además de la lectura y el almacenaje de distintos libros electrónicos en un único aparato, funciones asociadas como anotaciones, calendarios, etc. También se pueden usar dispositivos diseñados específicamente para la lectura de eBooks, como son $\mathrm{Mi}$ crosoft Reader, Palm Reader, DX Reader, Mobipocket, y los suministrados por Gemstar.

Cada fabricante intenta mejorar la usabilidad, accesibilidad y funciones de los eBooks, para acostumbrar a los usuarios finales a su formato.

Con estos lectores la mejora en las prestaciones es obvia, dado que disponen de una pantalla mayor, más capacidad de almacenaje de títulos, y más duración de la batería. $T \& F$ ofrece también sus contenidos para DX Reader (en caso de compra del eBook). El resto de las principales editoriales lo suministran en formato pdf (incluida $T \& F$ ).

En general todos los editores prevén en sus licencias el acceso ilimitado a múltiples usuarios, mediante autenticación por IP o bien por usuario y contraseña (algunas de ellos también por autenticación en Athens o Shibboleth).
También posibilitan acceder con usuario/contraseña - exceptuando Royal Society of Chemistry eBooks, John Wiley y World Scientific eBooks, que únicamente ofrecen autenticación por reconocimiento de la dirección IP del usuario.

Además, $C A B$ eBooks y $T \& F$ permiten autenticación a través de Athens; Cambridge University Press a través de Athens y Shibboleth; y MedicinesComplete a través de Athens y Single Sign On.

Los formatos en que se suministran las estadísticas de uso son xls, csv y html, algunos de ellos siguiendo ya los criterios Counter (por ejemplo, Karger, ScienceDirect, RSCPublishing, Springer, $T \& F$ y Wiley). Sólo World Scientific eBooks no ofrece, por el momento, ningún tipo de estadísticas de uso, pero se prevé que lo hará muy pronto.

\subsection{Temas y colecciones}

La temática tratada para el ámbito académico y científico-técnico es muy variada $y$, para acotar su análisis podríamos establecer una sencilla clasificación clásica:

- ciencia y tecnología,

- ciencias sociales y humanidades.

En la primera categoría destacan editores como Springer, Elsevier (a través de ScienceDirect), $C A B$, Karger, RSCPublishing y Wiley, mientras que en la segunda hay que mencionar las colecciones de Oxford University Press y Cambridge University Press.

Caso aparte es la editorial $T \& F$, que cubre un amplísimo espectro de materias.

Un análisis más detallado de los principales editores internacionales nos ofrece la siguiente información:

\section{CAB eBooks}

Sus 240 títulos pueden agruparse en 6 temas: agricultura, veteri- 
naria, ciencias medioambientales, ciencias alimentarias, turismo y tiempo libre, y botánica.

\section{Elsevier}

Suministra 4.000 eBooks en 20 colecciones científico-técnicas a través de la plataforma ScienceDirect.

\section{Karger}

Ciencias biomédicas, aplicación clínica e investigación biomédica.

\section{MedicinesComplete (RPS Publis- hing)}

Toxicología, análisis químicos, interacciones, herbología, medicina y dietética.

\section{RSC eBooks (Royal Society of Chemistry)}

Química.

\section{Wiley}

Medicina, física, química y materiales, informática, ciencias medioambientales, astronomía, medicina, y gestión económica y empresarial.

\section{Springer}

Es el mayor editor de eBooks para el mercado STM (scientifical, technical, medical). Total 5.000 eBooks (3.500 de ellos en inglés y 1.500 en alemán).

\section{Cambridge University Press}

Esta editorial presenta cinco colecciones muy específicas: Cambridge companions online; Shakespeare survey online; Historical statistics of the USA; Lectrix (textos para estudiantes de latín y griego); Orlando (Women's Literature);

\section{Taylor \& Francis}

Es la editorial que ofrece más variedad de disciplinas y de títulos: humanidades, ciencias sociales, psicología, ciencia y tecnología, derecho...

\section{Sage}

Se ha especializado en manuales y obras de referencia.

\subsection{DRM's (digital rights management)}

La DRM (digital rights management), es decir, la gestión de derechos digitales se refiere a la copia, envío, descarga, compartición e impresión de contenidos de los eBooks. En general son pocos los editores que indican restricciones en cuanto a DRM. Únicamente lo advierten confiando en el buen uso que se hará de los contenidos por parte de los usuarios (aunque se reservan el derecho a emprender acciones legales en caso de mal uso).

Ese es el caso de $C A B$ eBooks, que permite el acceso a múltiples usuarios y no establece limitaciones específicas de DRM más que los usuarios los respeten. En el mismo grupo se encuentran las editoriales Elsevier eBooks, Karger, RSC Publishing (Royal Society of Chemistry eBooks), Springer, Wiley y World Scientific eBooks.

En cambio, Cambridge University Press sí manifiesta restricciones de DRM, pero sólo las estipuladas con el autor en cuanto a copia, pegado o impresión de contenido. $T \& F$ reivindica limitaciones claras para la impresión y copia de contenidos y sólo permite el acceso de un usuario simultáneo a un título, previendo la opción de reserva y aviso a los usuarios que no pueden acceder al mismo tiempo al eBook.

\section{Agregadores de eBooks}

Los agregadores ofrecen un servicio de acceso a múltiples eBooks publicados por diferentes editores a través de una misma plataforma. De entre todos los existentes, en este apartado se analizarán las características de tres de los más usados y que más han evolucionado en los últimos años. El análisis se centrará de forma muy especial en la plataforma MyiLibrary que a partir de este año 2008 ofrecerá el acceso a sus eBooks junto a revistas electrónicas de diferentes editores a través de Swets.
Los principales agregadores de eBooks del mercado son:

- NetLibrary

- Ebrary-eLibro

- MyiLibrary

\section{NetLibrary}

En noviembre de 2005 este sistema de $O C L C$ fue el primero en suministrar libros-e, contando ahora con más de 100.000 títulos. Ofrece resúmenes automáticos asociados a los contenidos buscados, además de alternativas de búsqueda en caso de haber escrito un término incorrectamente o bien para refinar más la búsqueda. NetLibrary guarda las búsquedas realizadas y permite su personalización, incluye todo tipo de autenticación y EZproxy (integrado en sistemas de gestión de bibliotecas). Los temas de las colecciones de NetLibrary son:

Economía y finanzas; Estudios y carreras (principalmente guías); Recursos para profesores; Ingeniería; Ciencias sociales; Temas de actualidad (Hot topics); Tecnologías de la información; Medicina y crecimiento personal.

Resumiendo, NetLibrary ofrece libros electrónicos sobre todos los temas, incluyendo obras de referencia, informática, arte, ficción, etc. Es notable su contenido académico.

Cuenta con Title Select, una opción que permite configurar el menú de NetLibrary con los principales títulos disponibles o no en la institución.

\section{http://www.netlibrary.com/}

\section{Ebrary eLibro}

Distribuye 20.000 títulos para eLibro, y permite a las bibliotecas guardar y distribuir electrónicamente contenidos de sus colecciones especiales o reservadas.

Las colecciones de eLibro son de ciencias de la salud, ciencias económicas y administración de 


\section{Shibboleth}

1. En hebreo significa "mazorca o espiga" y también "arroyo o torrente". Según la Biblia la pronunciación de esta palabra fue utilizada por la tribu de los gileditas para distinguir a sus enemigos

los efraimitas, cuyo dialecto carecía del sonido sh.

Actualmente el significado es más amplio, y un shibboleth es cualquier cosa común a un grupo, que identifica a todos sus miembros (un acento, una característica física, una costumbre, un

rasgo de personalidad, compartición de una vivencia...).

2. Shibboleth es un software gratuito y de código abierto para gestionar las autorizaciones de acceso individual a recursos de acceso restringido o de pago, preservando la intimidad dentro de una organización.

http://shibboleth.internet2.edu/

\begin{tabular}{|c|} 
Athens \\
Sistema británico que permite a los usuarios tener una \\
identificación única para acceder a muchos recursos web distintos. \\
Reduce la carga administrativa para los bibliotecarios y gestores \\
de información, y ayuda a los proveedores de contenidos a \\
maximizar su mercado alcanzando una mayor audiencia. Athens \\
usa Shibboleth. \\
http://www.athensams.net/ \\
Athens es un servicio de Eduserv, Reino Unido. \\
http://www.eduserv.org.uk/
\end{tabular}

conocidos a nivel mundial ya descritos. Además dispone de acceso exclusivo a publicaciones de tipo intergubernamental que provienen de la actividad de instituciones mundiales como son la Agencia Internacional de Energía Atómica, la Organización Mundial de la Salud y la Organización Marítima Internacional, entre otras.

A través de MyiLibrary es posible la adquisición de eBooks por compra y por suscripción, tanto de paquetes enteros de colecciones, como eligiendo títulos individuales.

Dispone además de autenticación por IP, Shibboleth a través de Athens, usuario y contraseña, URL de referencia o EZ proxy.

MyiLibrary abarca todas las opciones de búsqueda existentes en el mercado. El usuario final puede personalizarse la cuenta a través de un mensaje de bienvenida e introducir el logo de su institución en la misma.

Dispone de más de 120.000 títulos de medicina, arquitectura, astronomía, biología, economía y empresas, además de tener un colección completa en español y otra en portugués (ambas incluyen temas de economía, ingeniería, ciencias, etc.). ELibro permite el acceso de múltiples usuarios, búsqueda avanzada a través de InfoTools (herramienta específica para eLibro), integración con sistemas de gestión de bibliotecas y recursos digitales, entre otras.

http://www.ebrary.com/

\section{MyiLibrary}

Es la solución en línea ofrecida por Ingram Digital Group's y distribuida también por Swets.

La siguiente tabla nos ofrece una comparativa de las funcionalidades y características de las tres plataformas:

MyiLibrary distribuye títulos de los editores de eBooks más re-

\begin{tabular}{|l|c|c|c|}
\hline & MyiLibrary & NetLibrary & eBrary \\
\hline No de títulos & 125.000 & 150.000 & 130.000 \\
\hline Athens & sí & sí & sí \\
\hline IP & sí & sí & sí \\
\hline Shibboleth & no & sí & no \\
\hline Usuario/contraseña & sí & sí & sí \\
\hline URL de referencia/EZproxy & sí & sí & sí \\
\hline Copia/impresión & sí & sí & sí \\
\hline Pegado & sí & no & sí \\
\hline DRM & sí & sí & sí \\
\hline Counter compliant & sí & no & no \\
\hline Counter book reports & no & no & no \\
\hline Disponibles online & sí & sí & sí \\
\hline Disponibles para envío al mail & sí & no & sí \\
\hline Disponibles en formato sushi & no & no & no \\
\hline
\end{tabular}

Tabla 1. Comparación de tres plataformas agregadoras de eBooks Fuente: JISC Collections. Academia database assessment tool, 2008 
finanzas, química y física, informática, criminología, educación, ingeniería, filosofía, psicología y ciencias sociales, veterinaria, enfermería, derecho y humanidades entre otros.

Es una plataforma fácil de integrar desde OPACs y provee registros Marc 21.

Tras el acuerdo al que llegaron en 2007 Swets y MyiLibrary, los clientes de ambos proveedores disponen de las revistas electrónicas y de los eBooks en una misma plataforma y con un único punto de acceso, conservando las diferentes modalidades de adquisición.

\section{http://www.myilibrary.com}

\section{Tendencias de futuro}

Según el International Digital Publishing Forum ${ }^{2}$, en 2002 las ventas de eBooks en los Estados Unidos fueron de 5,8 millones de US\$. Para 2007 resultaron ya de 31,8 millones de US\$. Estas cifras corresponden a las ventas al mayor, con descuento para las librerías. Si tomáramos el precio de venta al público los valores deben duplicarse. Además haría falta añadir a los pequeños editores no asociados a la American Publishers Association, cuyas ventas según algunos expertos representan otro $20 \%$.

Este incremento espectacular nos ofrece una certeza: a lo largo de su corta historia los eBooks disponen de un fortísimo apoyo.

Las mejoras en cuanto a la accesibilidad y los dispositivos y programas para leer un ebook han ayudado a este incremento. No sólo por este motivo, sino que la posibilidad de acceder a una cantidad casi ilimitada de información, funciones multimedia, actualización constante, enlazar con otros recursos..., hacen de los eBooks uno de los recursos más atractivos para estudiantes, investigadores y bibliotecarios.

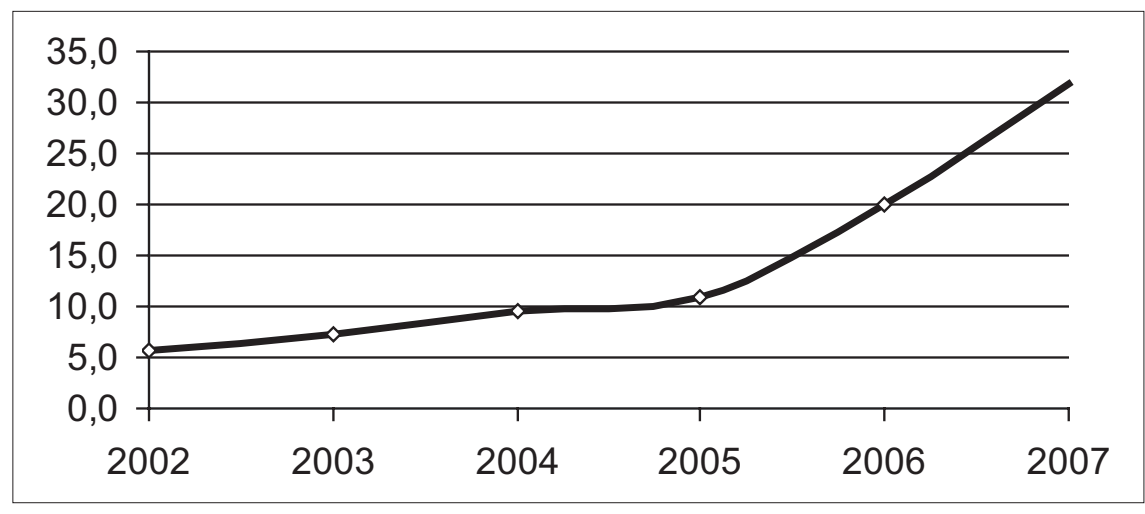

Gráfico 2. Evolución de las ventas de eBooks al mayor en EUA (en millones de US\$) Fuente: Estadísticas del IDPF y de la AAP.

En resumidas cuentas, un eBook dispone del potencial de complementar la experiencia de usar las nuevas tecnologías a la par que la de una buena lectura ${ }^{3}$.

El éxito de los eBooks está asegurado. En el ámbito de la investigación, poder acceder al contenido de eBooks y revistas electrónicas mediante una única plataforma y ser gestionados en la misma, será un avance más tanto para bibliotecarios y gestores documentales como para investigadores.

Formamos parte de la era de Google, pero pensemos especialmente en la generación que viene. Ellos ya están preparados y esperan encontrar recursos acordes con sus necesidades ¿está su biblioteca preparada para ellos?

\section{Notas}

1. Harris, Siân. "Revolutionising background research". En: Research information, 2007, April/May, n. 29.

2. IDPF. Digital book 2008. Industry statistics, US Trade Wholesale Electronic Books Sales 14/04/2009.

http://www.idpf.org/doc_library/industrystats. htm

3. Towle, Gemma; Dearnley, James A.; McKnight, Cliff. "Electronic books in the 2003-2005 period: some reflections on their apparent potential and actual development". En: Publishing research quarterly, 2007, v. 23, n. 2, Springer Verlag.

\section{Bibliografía y recursos consultados}

Algenio, Emilie; Thompson-Young, Alexia.
"Licensing e-books: the god, the bad, and the ugly". En: Journal of library administration, 2005 , v. 42 , n. $3 / 4$.

Anuradha, K. T.; Usha, H. S. "E-books access models: an analytical comparative study". En: Electronic library. Emerald Group Publishing Limited, 2006, v. 24, n. 5.

Beebe, Linda. "Library/vendor relations: the APA experience". En: Journal of library administration, 2006, v. 44, n. $3 / 4$.

Crestani, Fabio; Landoni, Monica; Melucci, Máximo. "Appearance and functionality of electronic books". En: International journal on digital libraries, 2006, v. 6, n. 2, Springer Verlag.

Gibbons, Susan. "Ebooks: Some concerns and surprises". En: Portal - Libraries and the acade$m y, 2001$, v. 1, n. 1 .

Gibson, Matthew; Ruotolo, Christine. "Beyond the Web: TEI, the digital library, and the ebook revolution". En: Computers and the humanities, 2003, v. 37, pp. 57-63. Kluwer Academic Publishers.

Mort, David. "Online information drives growth". En: Research information, 2007, August/September, n. 31

Rebiun. Indicadores estadísticos 2001-2007, 01/07/2008

http://www.rebiun.org/cuestionarios/indicadores/ indicadores_main.asp\#\#

Safley, Ellen. "Demand for E-books in an academic library". En: Journal of library administration, 2006, v. 45, n. $3 / 4$.

Van Gisbergen, Maxim. Swets ebook guide 2008, Lisse, Netherlands, 2008 Jul 02.

Juan-Carlos Martín, Swets, Country manager

jmartin@es.swets.com

Emma Pivetta, Swets, Electronic product manager

epivetta@es.swets.com 